\title{
Fermion spectral function in hot strongly interacting matter from the functional renormalization group
}

\author{
Ziyue Wang ${ }^{1, *}$ and Lianyi $\mathrm{He}^{1,2}$ \\ ${ }^{1}$ Physics Department, Tsinghua University, Beijing 100084, China \\ ${ }^{2}$ State Key Laboratory of Low-Dimensional Quantum Physics, Tsinghua University, \\ Beijing 100084, China
}

(Received 9 October 2018; published 29 November 2018)

\begin{abstract}
We present the first calculation of the fermion spectral function at finite temperature in the quark-meson model in the framework of the functional renormalization group. We compare the results in two truncations; after first evolving the flow equation of effective potential, we investigate the spectral function either by taking the IR values as input to calculate one-loop self-energy or by taking the scale-dependent values as input to evolve the flow equation of the fermion two-point function. The latter one is a selfconsistent procedure in the framework of the functional renormalization group. In both truncations, we find a multipeak structure in the spectral function, indicating quark collective excitations realized in terms of the Landau damping. However, in contrast to the fermion zero mode in the one-loop truncation, we find a fermion soft mode in the self-consistent truncation, which approaches the zero mode as the temperature increases.
\end{abstract}

DOI: 10.1103/PhysRevD.98.094031

\section{INTRODUCTION}

The QCD phase transitions at finite temperature and density provide deep insight into the strong interacting matter created in high-energy nuclear collisions and compact stars. The properties of the quark gluon plasma (QGP) phase near the critical temperature $\left(T_{c}\right)$ have acquired much interest; the heavy-ion collisions have suggested that the QGP matter is an ideal fluid [1,2], indicating that the created matter is a strongly coupled system. The spectral properties of the quark and hadron in this strongly interacting matter are of fundamental importance for identifying the relevant degrees of freedom (d.o.f.) in the equation of state and respective transport properties.

Whether quark can be described by well-defined quasiparticles has long been investigated. Quasiparticles correspond to peaks with a small width in the spectral function with relevant quantum numbers. Quenched lattice QCD simulation indicates the existence of the quasiparticles of quarks with a small decay width [3-5]. Finite-temperature gauge theory with the Dyson-Schwinger equation also predicts quasiparticle properties of quarks [6,7]. At high temperature, where the hard thermal loop approximation

*zy-wa14@mails.tsinghua.edu.cn

Published by the American Physical Society under the terms of the Creative Commons Attribution 4.0 International license. Further distribution of this work must maintain attribution to the author(s) and the published article's title, journal citation, and DOI. Funded by SCOAP . applies, quarks still have some collective excitation known by the normal quasiquark and the plasmino branches in the spectral function [8-10]. It has also been investigated that quarks in the QGP phase can be described within a quasiparticle picture with a multipeak spectral function [11-13], whenever the interaction is mediated by scalar, pseudoscalar, vector, and axial-vector mesons, which may exist as bosonic excitations in the QGP phase [13].

In the vicinity of $T_{c}$ of the chiral phase transition, nonperturbative effects are important, and one may expect that the quark spectral functions will possess novel properties when nonperturbative methods are adopted. In this paper, we employ the functional renormalization group (FRG) [14-19] approach to the quark-meson model. As a nonperturbative method, the FRG enables us to incorporate fluctuation effects beyond mean field theory; see Refs. [14]. The self-consistent treatment of fluctuations is important for the understanding of physics near a phase transition. Since the FRG allows a description of scale transformation, it provides deep insight into the system where scale dependence plays a crucial role. To calculate the spectral function in the usually used imaginary time formalism with the FRG, an analytical continuation is required to bring the imaginary time in the Euclidean two-point function at finite temperature to the real time in the Minkowski space [20-26]. This method has been applied to the study of realtime observables such as shear viscosity [26] and soft modes [27] near the QCD critical point. The consistent investigation of the spectral function in the framework of the FRG has been applied to various systems, including the 
meson spectral function in a chiral phase transition [23,24], quark spectral function in vacuum [28], and meson spectral function in a pion superfluid system [29].

When the FRG is put to use to investigate the quark spectral function, the most crucial difference is that one takes into account the scale dependence of the meson masses, and hence the thresholds of each decay, creation, and scattering channel. The scale dependence is a highorder effect and brings about a difference in spectral function, mainly in the following three aspects. First, it gives rise to novel structures in the imaginary part and real part of the self-energy. Second, the Landau damping, which is the dominant effect at high temperature, is forbidden at low energy when considering the scale dependence of the meson masses and leads to more peaks in the low-energy region at high temperature. Third, it is found that a fermion zero mode starts to appear when temperature is comparable to meson mass [13], which also originates from the Landau damping effect. However, when the FRG is adopted, this zero mode becomes a soft mode and approaches the origin when temperature increases.

We organize the paper as follows. The FRG flow equations for the effective potential and the two truncations to calculate the quark self-energy are derived in Sec. II. The procedure to solve the flow equations and the numerical results are presented in Sec. III. We summarize in Sec. IV.

\section{FLOW EQUATIONS AND TRUNCATION}

As a low-energy effective model, the quark-meson model comes from the partial bosonization of the fourfermion interaction model and exhibits many of the global symmetries of QCD. It is widely used as an effective chiral model to demonstrate the spontaneous chiral symmetry breaking in vacuum and its restoration at finite temperature and density [30-32]. Here, we take the two-flavor version of the model with pseudoscalar mesons $\pi$ and scalar meson $\sigma$ as the dominant meson d.o.f. at an energy scale up to $\Lambda \approx 1 \mathrm{GeV}$. The Euclidean effective action of the model at finite temperature $T$ and density $\mu$ is given as

$$
\begin{aligned}
\Gamma= & \int_{x}\left[\bar{\psi}\left(\not \partial-\gamma_{0} \mu\right) \psi+g \bar{\psi}\left(\sigma+i \gamma_{5} \vec{\tau} \cdot \vec{\pi}\right) \psi\right. \\
& \left.+\frac{1}{2}\left(\partial_{\mu} \phi\right)^{2}+U\left(\phi^{2}\right)-c \sigma\right]
\end{aligned}
$$

where the abbreviation $\int_{x}$ stands for $\int_{0}^{\beta} d x_{0} \int d^{3} x$ with the inverse of temperature $\beta=1 / T$ and $\vec{\tau}$ are the Pauli matrices in flavor space. The Yukawa coupling is chosen as $g=3.2$ to fit the quark mass in vacuum. The fermion field $\psi$ and meson field $\phi$ are defined as $\psi=(u, d)$ and $\phi=\left(\sigma, \pi_{1}, \pi_{2}, \pi_{0}\right)$. The explicit chiral symmetry breaking term $-c \sigma$ corresponds to a finite current quark mass $m_{0}$.

Quantum and thermal fluctuations are of particular importance in the vicinity of a phase transition and are conveniently included within the framework of FRG. The core quantity in this approach is the averaged effective action $\Gamma_{k}$ at the renormalization group (RG) scale $k$ in Euclidean space, and its scale dependence is described by the flow equation [14-19]

$$
\dot{\Gamma}_{k}=\operatorname{Tr} \int_{p}\left[\frac{1}{2} G_{\phi, k}(p) \dot{R}_{\phi, k}(p)-G_{\psi, k}(p) \dot{R}_{\psi, k}(p)\right],
$$

where $\dot{\Gamma}_{k}=\partial_{k} \Gamma_{k}$ and $\dot{R}_{k}=\partial_{k} R_{k}$. The symbol Tr represents the summation over all inner d.o.f. of mesons and quarks;

$$
\begin{aligned}
G_{\phi, k}(q) & =\left(\Gamma_{k}^{(2)}[\phi]+R_{\phi, k}(q)\right)^{-1}, \\
G_{\psi, k}(q) & =\left(\Gamma_{k}^{(2)}[\psi]+R_{\psi, k}(q)\right)^{-1}
\end{aligned}
$$

are the FRG-modified meson and quark propagators with the two-point functions $\Gamma_{k}^{(2)}[\phi]=\delta^{2} \Gamma_{k} / \delta \phi^{2}$ and $\Gamma_{k}^{(2)}[\psi]=$ $\delta^{2} \Gamma_{k} / \delta \psi \delta \bar{\psi}$ and the two regulators $R_{\phi, k}$ and $R_{\psi, k}$. The evolution of the flow from the ultraviolet limit $k=\Lambda$ to the infrared limit $k=0$ encodes, in principle, all the quantum and thermal fluctuations in the action. To suppress the fluctuations with momentum smaller than the scale $k$ during the evolution, an infrared regulator $R$ is introduced in the flow equation. At finite temperature and density where the Lorentz symmetry is broken, we employ the optimized regulator function, which is the three-dimensional analog of the 4-momentum regulator $[33,34]$. The bosonic and fermionic regulators are chosen to be

$$
\begin{aligned}
& R_{\phi, k}(p)=\vec{p}^{2} r_{B}(y), \\
& R_{\psi, k}(p)=\vec{\gamma} \cdot \vec{p} r_{F}(y)
\end{aligned}
$$

in momentum space with $y=\vec{p}^{2} / k^{2}$ and $r_{B}(y)=$ $(1 / y-1) \Theta(1-y) \quad$ and $\quad r_{F}(y)=(1 / \sqrt{y}-1) \Theta(1-y)$. The regulators $R_{\phi, k}$ and $R_{\psi, k}$ in the propagators $G_{\phi}$ and $G_{\psi}$ amount to having regularized 3-momenta $\vec{p}_{r}^{2}=$ $\vec{p}^{2}\left(1+r_{B}(y)\right)$ and $\vec{p}_{r}=\vec{p}\left(1+r_{F}(y)\right)$ for bosons and fermions, respectively. The three-dimensional regulators have the advantage of not introducing additional poles in the complex $\omega$ plane at a finite scale. Yet the disadvantage is breaking the space-time symmetry during the evolution of the flow equation; this effect slightly remains in the infrared result. It has been discussed in Ref. [20] that the peak position is not affected; thus the approach is still reliable.

To derive the meson and quark propagators $G_{\phi, k}$ and $G_{\psi, k}$, we expand the effective potential around the mean field $\langle\sigma\rangle_{k}$, which describes the chiral symmetry breaking, and introduce the chiral invariant $\rho_{k}=\langle\sigma\rangle_{k}^{2}$. The RG-modified propagators of mesons and the quark are 


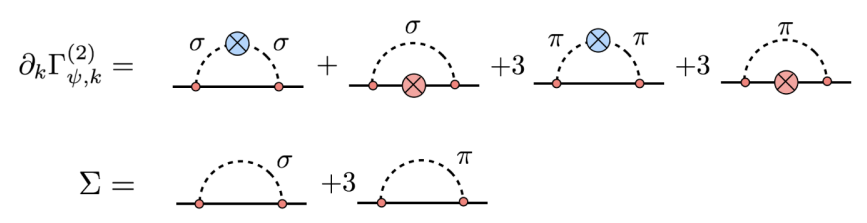

FIG. 1. The diagrammatic presentation of self-energy in two truncations: $\partial_{k} \Gamma_{\psi, k}^{(2)}$ is the flow equation for the fermion two-point function in truncation $\mathrm{A}$, and $\Sigma$ is the one-loop self-energy in truncation B.

$$
\begin{aligned}
G_{\phi, k}^{-1} & =p_{0}^{2}+\vec{p}_{r}^{2}+m_{\phi, k}^{2}, \\
G_{\psi, k}^{-1} & =-\gamma_{0}\left(i p_{0}+\mu\right)+\vec{\gamma} \cdot \vec{p}+m_{f, k},
\end{aligned}
$$

where $\phi=\sigma, \pi$, the meson mass are given by $m_{\sigma, k}^{2}=2 U^{\prime}+$ $4 \rho_{k} U^{\prime \prime}$ and $m_{\pi, k}^{2}=2 U^{\prime} . U^{\prime}$ and $U^{\prime \prime}$ are the first and second order derivative of the effective potential with respect to $\rho$. And the quark mass is $m_{f, k}=g\langle\sigma\rangle_{k}$.

Assuming uniform field configurations, the integral over space and imaginary time becomes trivial, and the effective action $\Gamma_{k}=\beta V U_{k}$ is fully controlled by the potential $U_{k}$ with $V$ and $\beta$ being the space and time regions of the system. The flow equation $U_{k}$ hence comes directly from that of $\Gamma_{k}$, namely, $\partial_{k} U_{k}=$ $(T / V) \partial_{k} \Gamma_{k}$. The flow equation of the effective potential is then calculated by

$$
\partial_{k} U_{k}=\frac{1}{2} J_{\phi}\left(E_{\sigma, k}\right)+\frac{3}{2} J_{\phi}\left(E_{\pi, k}\right)-N_{c} N_{f} J_{\psi}\left(E_{\psi, k}\right),
$$

with $J_{\phi}$ and $J_{\psi}$ being one-loop threshold functions; the explicit expressions are presented in the Appendix by Eq. (A2), and the energies are given by $E_{\phi, k}=\sqrt{k^{2}+m_{\phi, k}^{2}}$ and $E_{\psi, k}=\sqrt{k^{2}+m_{\psi, k}^{2}}$.

In the following, we are going to present two truncations to calculate the self-energy and the spectral function. In both truncations, we first evolve the flow of the effective potential and then take the masses at IR minimum $\rho_{0}$ of the potential as input to calculate the self-energy. In truncation A, we take scale-dependent curvature masses $m_{\sigma, k}=$ $\sqrt{2 U_{k}^{\prime}\left(\rho_{0}\right)+4 \rho_{0} U_{k}^{\prime \prime}\left(\rho_{0}\right)}$ and $m_{\pi, k}=\sqrt{2 U_{k}^{\prime}\left(\rho_{0}\right)}$ as input to integrate the flow of the two-point function. While in truncation $\mathrm{B}$, we take the IR curvature masses $m_{\sigma, 0}=$ $\sqrt{2 U_{0}^{\prime}\left(\rho_{0}\right)+4 \rho_{0} U_{0}^{\prime \prime}\left(\rho_{0}\right)}$ and $m_{\pi, 0}=\sqrt{2 U_{0}^{\prime}\left(\rho_{0}\right)}$ to directly calculate the one-loop self-energy of quark. The diagrammatic description is presented in Fig. 1.

\section{A. Truncation A}

In truncation A, we first evolve the flow of the effective potential and prepare the scale-dependent meson masses as input. We then integrate the flow equation of the twopoint function to obtain the self-energy in the infrared limit.
The flow equation of the two point function of a $u$ quark with certain color is given by,

$$
\begin{aligned}
\partial_{k} \Gamma_{\bar{u} u}^{(2)}(p)= & -g^{2} \tilde{\partial}_{k} \int_{q}\left[G_{\sigma}(q-p) G_{u}(q)\right. \\
& \left.+3 G_{\pi}(q-p)\left(i \gamma_{5}\right) G_{u}(q)\left(i \gamma_{5}\right)\right],
\end{aligned}
$$

where $\tilde{\partial}_{k}$ is the derivative of RG-scale $k$, and $\tilde{\partial}_{k}$ acts only onto the regulators $R_{\phi, k}$ and $R_{\psi, k}$ in the propagators. In this work, we consider only the spectral function at zero external momentum $\vec{p}=0$. In the ultraviolet, the Euclidean inverse quark propagator at $\vec{p}=0$ at the IR expansion point is

$$
G_{E, \Lambda}^{-1}\left(i p_{0}\right)=\Gamma_{\bar{u} u, \Lambda}^{(2)}\left(i p_{0}\right)=-\gamma^{0}\left(i p_{0}+\mu\right)+g\langle\sigma\rangle .
$$

The inverse propagator at scale $k$ is then an integral of the flow equation two-point function from the ultraviolet $k=\Lambda$ down to $k$,

$$
\begin{aligned}
G_{E, k}^{-1}\left(i p_{0}\right)= & \Gamma_{\bar{u} u, k}^{(2)}\left(i p_{0}\right)=-\gamma^{0}\left(i p_{0}+\mu\right)+g\langle\sigma\rangle \\
& +\int_{\Lambda}^{k} \partial_{k} \Gamma_{\bar{u} u}^{(2)}\left(i p_{0}\right) d k .
\end{aligned}
$$

Hence, the Euclidean inverse propagator can be written in terms of $k$-dependent self-energy $G_{E, k}^{-1}\left(i p_{0}\right)=\Gamma_{\bar{u} u, k}^{(2)}\left(i p_{0}\right)=$ $-\gamma^{0}\left(i p_{0}+\mu\right)+g\langle\sigma\rangle+\Sigma_{k}\left(i p_{0}\right)$, with the initial condition $\Sigma_{\Lambda}\left(i p_{0}\right)=0$. The scale-dependent self-energy in Euclidean space is thus

$$
\Sigma_{k}\left(i p_{0}\right)-\Sigma_{\Lambda}\left(i p_{0}\right)=\int_{\Lambda}^{k} \partial_{k} \Gamma_{\bar{u} u}^{(2)}\left(i p_{0}\right) d k .
$$

At ultraviolet limit $k=\Lambda$, no fluctuation is included, and $\Sigma_{\Lambda}\left(i p_{0}\right)=0$ agrees with the bare propagator. As the scale is lowered, quantum fluctuations are included, contributing to the self-energy of the quark. The spectral function is a realtime quantity that requires analytical continuation to bring imaginary time to real time,

$$
G_{R}^{-1}(\omega)=-G_{E}^{-1}\left(i p_{0} \rightarrow \omega+i \eta\right) .
$$

The inverse retarded propagator is $G_{R, k}^{-1}(\omega)=\gamma^{0}(\omega+\mu+i \eta)-$ $g\langle\sigma\rangle-\Sigma_{R, k}(\omega)$, and the corresponding retarded self-energy is

$$
\Sigma_{R, k}(\omega)=\int_{\Lambda}^{k} \partial_{k} \Gamma_{\bar{u} u}^{(2)}(\omega+i \eta) d k
$$

The quark propagator at zero momentum $\vec{p}=0$ can be decomposed into the positive energy part and negative energy part,

$$
G_{R, k}(\omega)=G_{+, k}(\omega) \Lambda_{+} \gamma^{0}+G_{-, k}(\omega) \Lambda_{-} \gamma^{0},
$$

with projection operators $\Lambda_{ \pm} \equiv\left(1 \pm \gamma^{0}\right) / 2$ acting onto spinors of which the chirality is equal to $(+)$ or opposite $(-)$ the 
helicity. We call the + (-) sector the "quark" ("antiquark") sector. Hence, the propagators for positive and negative energy parts are

$$
\begin{aligned}
G_{ \pm, k}(\omega) & =\frac{1}{2} \operatorname{Tr}\left[G_{R, k}(\omega) \gamma^{0} \Lambda_{ \pm}\right] \\
& =\left[\omega+i \eta+\mu \mp m_{f}-\Sigma_{ \pm, k}(\omega)\right]^{-1},
\end{aligned}
$$

with

$$
\Sigma_{ \pm, k}(\omega)=\frac{1}{2} \operatorname{Tr}\left[\Sigma_{R, k}(\omega) \gamma^{0} \Lambda_{ \pm}\right]
$$

We focus here on the self-energy of the quark sector. The flow equation is given by

$$
\partial_{k} \Sigma_{ \pm, k}(\omega)=\frac{1}{2} \operatorname{Tr}\left[\partial_{k} \Gamma_{\bar{u} u}^{(2)}(\omega) \gamma^{0} \Lambda_{ \pm}\right]
$$

which, after integral over the 3-momentum, Matsubara sum, and analytical continuation, has the structure

$\partial_{k} \Sigma_{+, k}(\omega)=g^{2}\left(J_{\psi \sigma}^{S}(\omega)+J_{\sigma \psi}^{S}(\omega)+3 J_{\psi \pi}^{P S}(\omega)+3 J_{\pi \psi}^{P S}(\omega)\right)$,

with $J_{\psi \sigma}^{S}, J_{\sigma \psi}^{S}, J_{\psi \pi}^{P S}$, and $J_{\pi \psi}^{P S}$ being the threshold functions presented in the Appendix. At vanishing quark number, the charge conjugation symmetry leads to a relation between $G_{ \pm}$,

$$
G_{+}(\omega)=-G_{-}^{*}(-\omega),
$$

However, the charge conjugation symmetry can be broken by the finite chemical potential. We limit our study to the case with $\mu=0$ and focus on the spectral function of the quark sector only. The spectral function is defined through the imaginary part of the retarded propagator $\rho_{k}(\omega)=-(1 / \pi) \operatorname{Im} G_{R, k}(\omega)$. This can be decomposed similarly, $\rho_{k}(\omega)=\rho_{+, k}(\omega) \Lambda_{+} \gamma_{0}+\rho_{-, k}(\omega) \Lambda_{-} \gamma_{0}$, with

$$
\begin{aligned}
\rho_{ \pm, k}(\omega) & =-\frac{1}{\pi} \operatorname{Im} G_{ \pm, k} \\
& =-\frac{1}{\pi} \frac{\operatorname{Im} \Sigma_{ \pm, k}(\omega)}{\left(\omega \mp m_{f}-\operatorname{Re} \Sigma_{ \pm, k}(\omega)\right)^{2}+\operatorname{Im} \Sigma_{ \pm, k}(\omega)^{2}} .
\end{aligned}
$$

\section{B. Truncation B}

In another truncation, we first evolve the flow equation of the effective potential and find the infrared quark mass $m_{f, k=0}(T, \mu)$ and meson mass $m_{\sigma, k=0}(T, \mu), m_{\pi, k=0}(T, \mu)$ at a certain temperature and density. We then take these quantities as input to calculate the self-energy of quark at one-loop order. This method has been discussed in Refs. [11-13], and here we take the FRG result as an input. In Euclidean space, the fermion self-energy is

$$
\begin{aligned}
\Sigma_{E}\left(i p_{0}\right)= & -g^{2} \int_{q}\left[G_{\psi}(q) G_{\sigma}(p+q)\right. \\
& \left.+3 G_{\psi}(q)\left(i \gamma^{5}\right) G_{\pi}(p+q)\left(i \gamma^{5}\right)\right] .
\end{aligned}
$$

The analytical continuation is taken by $\Sigma_{R}(\omega)=$ $\left.\Sigma_{E}\left(i p_{0}\right)\right|_{i p_{0} \rightarrow \omega+i \eta}$. Taking the energy projection as before, we have the self-energy of the quark and antiquark sector $\Sigma_{ \pm}(\omega)=\frac{1}{2} \operatorname{Tr}\left[\Sigma_{R}(\omega) \gamma^{0} \Lambda_{ \pm}\right]$. The self-energy has a contribution from the scalar channel and the pseudoscalar channel, where the interaction is mediated by the sigma meson and pion, respectively,

$$
\Sigma_{ \pm}(\omega)=\Sigma_{ \pm}^{S}(\omega)+3 \Sigma_{ \pm}^{P S}(\omega) .
$$

One may first deal with the imaginary part of the scalar channel

$$
\begin{aligned}
\operatorname{Im} \Sigma_{+}^{S}(\omega)= & -\frac{g^{2}}{2 \pi} \int_{0}^{+\infty} \frac{p^{2} d p}{E_{\sigma}}\left\{+\delta\left(\omega+E_{\sigma}+E_{f}\right)\left(1-\frac{m_{f}}{E_{f}}\right)\left[1+n_{b}\left(E_{\sigma}\right)-n_{f}\left(E_{f}\right)\right]\right. \\
& +\delta\left(\omega+E_{\sigma}-E_{f}\right)\left(1+\frac{m_{f}}{E_{f}}\right)\left[n_{b}\left(E_{\sigma}\right)+n_{f}\left(E_{f}\right)\right] \\
& +\delta\left(\omega-E_{\sigma}+E_{f}\right)\left(1-\frac{m_{f}}{E_{f}}\right)\left[n_{b}\left(E_{\sigma}\right)+n_{f}\left(E_{f}\right)\right] \\
& \left.+\delta\left(\omega-E_{\sigma}-E_{f}\right)\left(1+\frac{m_{f}}{E_{f}}\right)\left[1+n_{b}\left(E_{\sigma}\right)-n_{f}\left(E_{f}\right)\right]\right\} .
\end{aligned}
$$

The momentum integral can be carried out analytically, giving [12]

$$
\begin{aligned}
\operatorname{Im} \Sigma_{+}^{S}(\omega)= & -\frac{g^{2}}{64 \pi} \frac{\left(\omega+M_{+}\right)\left(\omega-M_{-}\right)}{\omega^{3}} \sqrt{\left(\omega^{2}-M_{+}^{2}\right)\left(\omega^{2}-M_{-}^{2}\right)}\left[\operatorname{coth} \frac{\omega^{2}+M_{+} M_{-}}{4 \omega T}+\tanh \frac{\omega^{2}-M_{-} M_{+}}{4 \omega T}\right] \\
& \times\left(\Theta\left(\omega^{2}-M_{+}^{2}\right)-\Theta\left(M_{-}^{2}-\omega^{2}\right)\right),
\end{aligned}
$$


where $M_{+}=m_{\sigma}+m_{f}$ and $M_{-}=\left|m_{\sigma}-m_{f}\right|$ and $\Theta(x)$ is the step function. The self-energy of the pseudoscalar channel can be obtain from that of the scalar channel by taking the substitution $m_{f} \rightarrow-m_{f}$ and $m_{\sigma} \rightarrow m_{\pi}$. The imaginary part of the self-energy is free from divergence, but the real part $\operatorname{Re} \Sigma^{R}(\omega)$ has an ultraviolet divergence that originates from the $T$-independent part $\Sigma_{T=0}^{R}(\omega) \equiv \lim _{T \rightarrow 0^{+}} \Sigma^{R}(\omega)$. The divergence can be removed by imposing the on-shell renormalization condition on the $T$-independent part of the quark propagator. This renormalization is carried out by using the subtracted dispersion relation; see Ref. [12]. Taking the zero-temperature limit of the imaginary part, one obtains

$$
\begin{aligned}
\operatorname{Im} \Sigma_{+, T=0}(\omega)= & -\frac{g^{2}}{32 \pi} \frac{\left(\omega+M_{+}\right)\left(\omega-M_{-}\right)}{\omega^{3}} \\
& \times \sqrt{\left(\omega^{2}-M_{+}^{2}\right)\left(\omega^{2}-M_{-}^{2}\right)} \\
& \times \frac{\omega}{|\omega|} \Theta\left(\omega^{2}-M_{+}^{2}\right) .
\end{aligned}
$$

The regularized real part $\operatorname{Re} \Sigma_{+, T=0}(\omega)$ in vacuum is then obtained using the subtracted dispersion relation

$\operatorname{Re} \Sigma_{+, T=0}(\omega)=\frac{(\omega-\alpha)^{n}}{\pi} \mathcal{P} \int_{-\infty}^{+\infty} d \xi \frac{\operatorname{Im} \Sigma_{+, T=0}^{R}(\xi)}{(\xi-\omega)(\xi-\alpha)^{n}}$,

where, in order to regularize the UV divergence, we have adopted the subtraction order $n=2$ and have considered the on-shell renormalization condition for the mass and wave function renormalization at the renormalization point $\alpha=m_{f}$. For details, we refer to Appendix A in Ref. [12]. The $T$-dependent part, $\Sigma_{T \neq 0}^{R}(\omega)=\Sigma^{R}(\omega)-$ $\Sigma_{T=0}^{R}(\omega)$ is free from divergence. The real part and imaginary part of the finite-temperature self-energy are related by the Krames-Kronig relation,

$$
\operatorname{Re} \Sigma_{+, T \neq 0}^{R}(\omega)=\mathcal{P} \int_{-\infty}^{+\infty} \frac{d z}{\pi} \frac{\operatorname{Im} \Sigma_{+, T \neq 0}^{R}(z)}{z-\omega} .
$$

Then, the vacuum part and finite-temperature part are combined to give the real part $\operatorname{Re} \Sigma_{+}=\operatorname{Re} \Sigma_{+, T=0}+$ $\operatorname{Re} \Sigma_{+, T \neq 0}$ The spectral functions of the quark and antiquark are then given by

$$
\begin{aligned}
\rho_{ \pm}(\omega) & =-\frac{1}{\pi} \operatorname{Im} G_{ \pm} \\
& =-\frac{1}{\pi} \frac{\operatorname{Im} \Sigma_{ \pm}(\omega)}{\left(\omega \mp m_{f}-\operatorname{Re} \Sigma_{ \pm}(\omega)\right)^{2}+\operatorname{Im} \Sigma_{ \pm}(\omega)^{2}} .
\end{aligned}
$$

\section{NUMERICAL METHOD AND RESULT}

To numerically solve the flow equations for the effective potential and the two-point functions, we adopt the grid

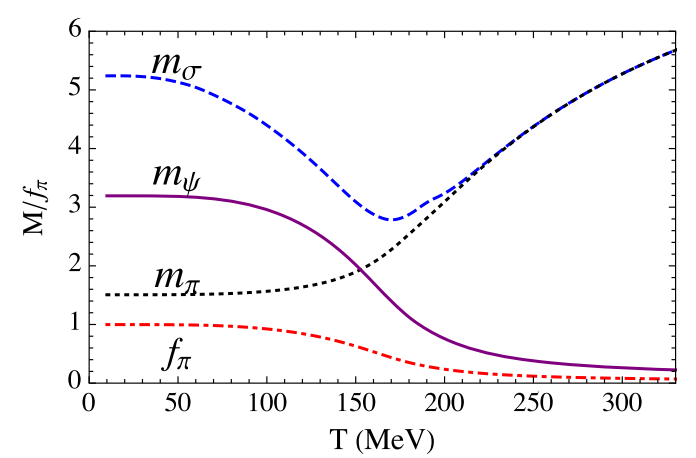

FIG. 2. Temperature dependence of chiral condensate and various masses.

method and assume the initial condition at the ultraviolet limit,

$$
U_{\Lambda}(\rho)=\frac{1}{2} m_{\Lambda}^{2} \rho+\frac{1}{4} \lambda_{\Lambda} \rho^{2},
$$

for the one-dimensional grid and for the quark self-energy

$$
\Sigma_{+, \Lambda}(\omega)=0
$$

During the process of integrating the flow equations from the ultraviolet limit to the infrared limit, the condensates $\langle\sigma\rangle_{k}$ are obtained by locating the minimum of the $k$-dependent effective potential $U_{k}$. Choosing the quark mass $m_{q}=300 \mathrm{MeV}$, pion mass $m_{\pi}=135 \mathrm{MeV}$, and pion decay constant $f_{\pi}=93 \mathrm{MeV}$ in vacuum, the corresponding initial parameters are $m_{\Lambda}^{2} / \Lambda^{2}=0.618, \lambda_{\Lambda}=1$, and $c / \Lambda^{3}=0.0025$ with the cutoff $\Lambda=900 \mathrm{MeV}$. In the numerical calculation, the infrared limit $k=0$ cannot be reached. Instead, the evolution of the flow equation is stopped at $k_{\mathrm{IR}}<10 \mathrm{MeV}$, where the condensate and coupling have reached a stable structure. By solving the flow equation of the effective potential Eq. (6), we obtain the dependence of chiral condensate and various masses on temperature, with critical temperature $T_{c} \sim 170 \mathrm{MeV}$. The result is presented in Fig. 2.

The imaginary part of the self-energy is proportional to the difference between the decay and creation rates of the quasiquark. Each of the four channels has a Dirac delta function, indicating the energy threshold for each process; see Eqs. (22), (A5), (A6), and (A7). One can give a physical interpretation to each of the channels:

(I) $\delta\left(\omega+E_{\phi}+E_{\psi}\right)$ describes the annihilation of a quasiquark with an on-shell free antiquark and boson,

(II) $\delta\left(\omega+E_{\phi}-E_{\psi}\right)$ is the decay process of a quasiquark state $\mathrm{Q}$, into an on-shell quark via a collision with a thermally excited boson and its inverse process $Q+b \leftrightarrow q$.

(III) $\delta\left(\omega-E_{\phi}+E_{\psi}\right)$ corresponds to a pair annihilation process between the quasiquark and a thermally 
excited antiquark with an emission of a boson into the thermal bath and its inverse process $Q+\bar{q} \leftrightarrow b$. Processes II and III are called Landau damping, which vanishes at $T=0$, as it involves thermally excited particles in the initial states. The Landau damping plays an important role in the spectral function as temperature rises and is closely related to the three-peak structure at high temperature. Processes II and III both cause a mixing between the quark and antiquark hole through coupling to a thermally excited boson as discussed in Ref. [12].

(IV) $\delta\left(\omega-E_{\phi}-E_{\psi}\right)$ is a decay of a quasiquark to an on-shell free quark and a boson,

In truncation $\mathrm{A}$, the real part and imaginary part of the self-energy are calculated separately. The real part is given by the principle value integral, while for the imaginary part of the two-point function, the integral over RG-scale $k$ only has a contribution from a few scales $k_{i}$ due to the appearance of aforementioned Dirac delta functions. The following structures are encountered in the integration of the imaginary part of the flow equation, where $g(k)=$ $\pm E_{\phi, k} \pm(\mp) E_{\psi, k}$ and $k_{i}$ are zero points of the delta function at a certain energy $\omega$, with $\omega+g\left(k_{i}\right)=0$ :

$$
\begin{aligned}
& \int_{\Lambda}^{0} f(k) \delta(\omega+g(k)) d k=-\sum_{i} \frac{f\left(k_{i}\right)}{\left|g^{\prime}\left(k_{i}\right)\right|}, \\
& \int_{\Lambda}^{0} f(k) \delta^{\prime}(\omega+g(k)) d k=\sum_{i}\left(\frac{f^{\prime}\left(k_{i}\right)}{g^{\prime}\left(k_{i}\right)}-\frac{f\left(k_{i}\right) g^{\prime \prime}\left(k_{i}\right)}{g^{\prime}\left(k_{i}\right)^{2}}\right) \\
& \quad \times \frac{1}{\left|g^{\prime}\left(k_{i}\right)\right|} .
\end{aligned}
$$

It is required that $g(k)$ is a continuously differentiable function with $g^{\prime}$ is nowhere zero. In the integral of the flow equation, $g(k)$ has certain points where the derivatives are zero, and the domain must be broken up to exclude the $g^{\prime}=0$ point. These $g^{\prime}\left(k_{i}\right)=0$ points are similar to the van Hove singularities in the density of states in condensed matter physics [35], $g(\omega)=\sum_{n} \int\left[d^{3} k /(2 \pi)^{3}\right] \delta\left(\omega-\omega_{n}(\vec{k})\right)=$ $\sum_{n} \int\left[d S_{\omega} /(2 \pi)^{3}\right]\left[1 /\left|\nabla \omega_{n}(\vec{k})\right|\right]$. The group velocity $\nabla \omega_{n}(\vec{k})$ vanishes at certain momenta, resulting in a divergent integrand. The divergence is integrable in three dimensions, and in lower dimensions, the van Hove singularity appears. The van Hove singularities have also attracted interest in high-energy physics [36-39]. In our case, the integral over $k$ is one dimensional and gives the divergence in the imaginary part at finite temperature. This divergence exists only in truncation A, when scale dependence of various masses is taken into consideration, and appears only when two conditions are satisfied at the same time, that $\omega+$ $\delta E\left(k^{*}\right)=0$ and $\delta E^{\prime}\left(k^{*}\right)=0$. The scale dependence of the meson mass also causes divergence in the real part of the selfenergy. In Fig. 3, we present the RG-scale dependence of the threshold for channels processes I to IV, $\pm E_{\phi, k} \pm E_{\psi, k}$

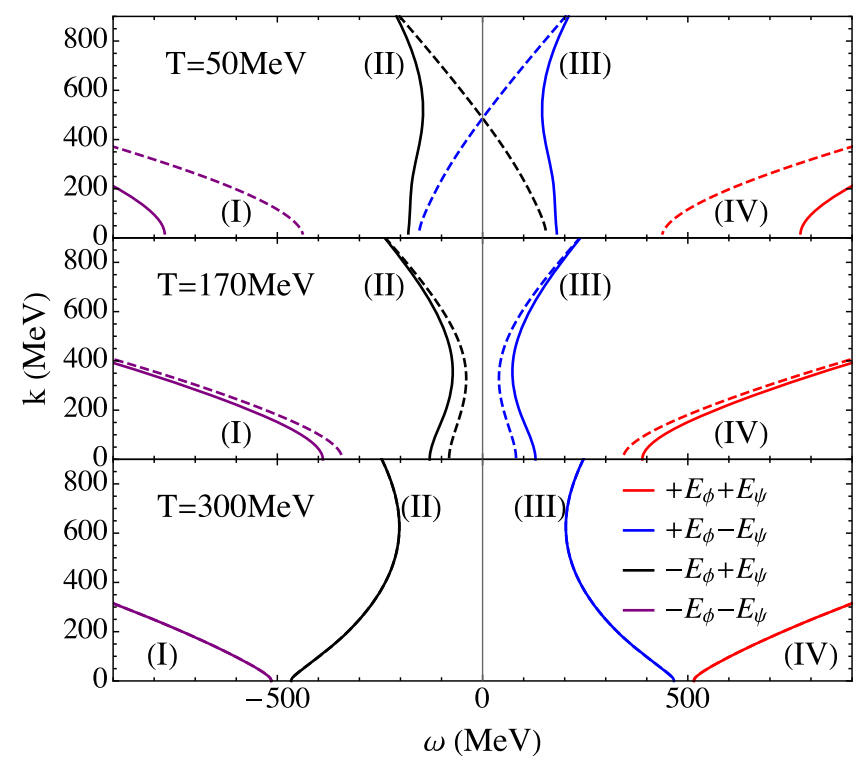

FIG. 3. The RG-scale dependence of the threshold for channels I to IV, $\pm E_{\phi, k} \pm E_{\psi, k}(\phi=\sigma, \pi)$, for three temperatures $T=50$, 170, $300 \mathrm{MeV}$.

( $\phi=\sigma, \pi)$, for three temperatures $T=50,170,300 \mathrm{MeV}$, with the dashed lines for pions and the solid line for sigma. For terms related to Landau damping, II and III, at certain $\omega$, the $k$ integral runs into points at which $\delta E^{\prime}\left(k^{*}\right)=0$, leading to the divergence in the imaginary part. At low temperature, when the Landau damping is suppressed, the divergence does not appear. In contrast, in truncation B, we take $m_{\phi, k=0}$ as input, and the imaginary part and real part are always finite after we remove the zero-temperature part.

We first present the spectral function of the quark sector at relatively low temperature, In Fig. 4, from top to bottom, are figures for the spectral function, the real part, and imaginary part of the self-energy, with the black solid line representing truncation $\mathrm{A}$ and the red dashed line representing truncation $\mathrm{B}$. The zero-temperature result can be found in Ref. [28], we have also found the same result as theirs. At $\mathrm{T}=50 \mathrm{MeV}$, the system is still in the chiral symmetry breaking phase, with quark mass $m_{f}=296 \mathrm{MeV}$ and meson mass $m_{\sigma}=477 \mathrm{MeV}$ and $m_{\pi}=140 \mathrm{MeV}$. In both truncations, we have delta peaks around the fermion mass, $\omega=315 \mathrm{MeV}$ for truncation A and $\omega=296 \mathrm{MeV}$ for truncation B. The peak structure in $\rho_{+}(\omega)$ emerges at the "quasipole" [12], which is defined as zero of the real part of the inverse propagator $\omega-m_{f}-\operatorname{Re} \Sigma_{+}(\omega)=0$, provided that the imaginary part is small enough at that point. In both truncations, the real part of the inverse propagator only has one quasipole, which is the cross point of $\omega-m_{f}$ (the blue dotted line) with $\operatorname{Re} \Sigma_{+}(\omega)$ in the figure. The position of the peaks in both truncations are slightly different, it is because of the difference in the methods of inclusion and subtraction of fluctuation. The Landau damping is still well suppressed, giving a small 


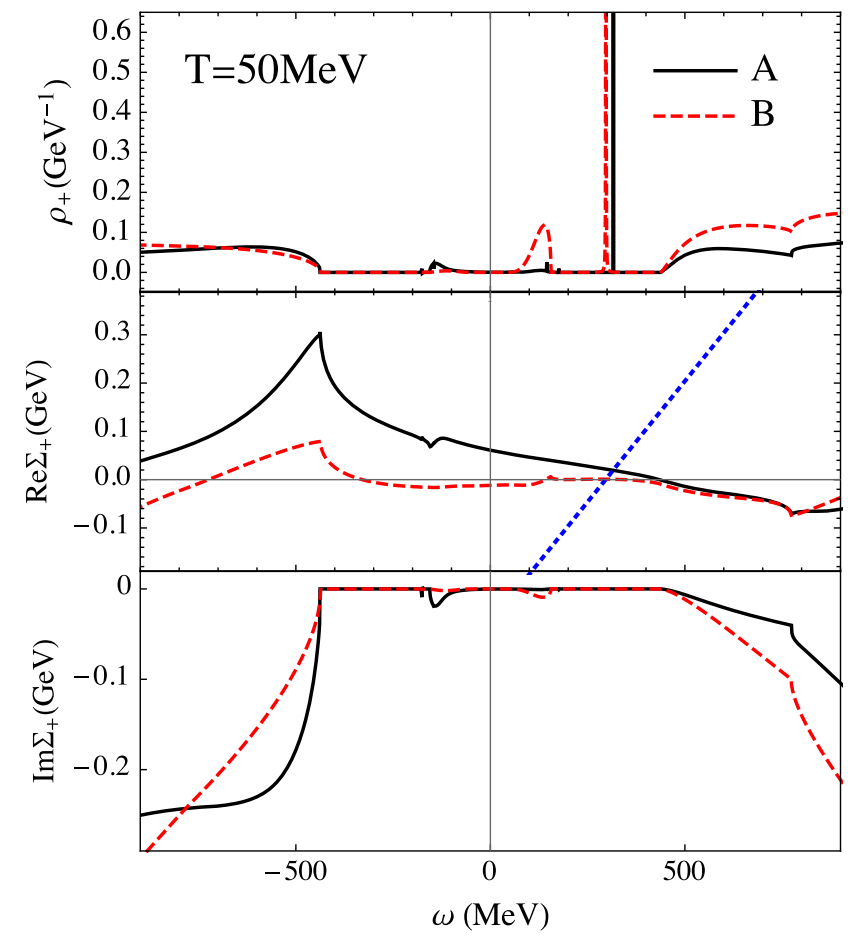

FIG. 4. Quark spectral function at $T=50 \mathrm{MeV}$ : from top to bottom are the spectral function and real and imaginary parts of the quark self-energy. The black solid line stands for truncation A, while the red dashed line stands for truncation $\mathrm{B}$. The blue dotted line represents $\omega-m_{f}$, with $m_{f}=296 \mathrm{MeV}$.

imaginary part and flat structures in the spectral function at low energy. At large energy, when $|\omega|>m_{\psi}+m_{\phi}$, the decay processes I and IV take place, giving a finite imaginary part and the continuous spectrum in both truncations, the difference comes from the two different ways of renormalizing the vacuum part.

The chiral phase transition takes place at the temperature about $T=170 \mathrm{MeV}$, with masses $m_{f}=130 \mathrm{MeV}$, $m_{\sigma}=259 \mathrm{MeV}$, and $m_{\pi}=212 \mathrm{MeV}$. At this temperature, the pion and sigma meson have not been degenerate yet. For truncation A, the threshold of various channels is presented in Fig. 3. In the scattering channel with the thermally excited boson (II) and the annihilation channel with the thermally excited antiquark (III), $\pm E_{\phi, k} \mp E_{\psi, k}$ has points where the derivative with the RG scale vanishes. This brings about divergence in the imaginary part and oscillation in the real part; see the black lines in Fig. 5. The imaginary part is discontinuous at the four van Hove singularities and is zero when $\omega \leq \mid \pm E_{\phi, k} \mp$ $\left.E_{\psi, k}\right|_{\min }$, where processes II and III are forbidden. $\omega-$ $m_{f}-\operatorname{Re} \Sigma_{+}(\omega)$ has several quasipoles in truncation $\mathrm{A}$, yet the spectral function has only one peak, when the imaginary part is small, $\omega=21 \mathrm{MeV}$, indicating a quasiparticle mode here. For other quasipoles, the imaginary parts are too large to form a peak, giving several bumps instead. In truncation $\mathrm{B}$, however, we have a quite different case: the

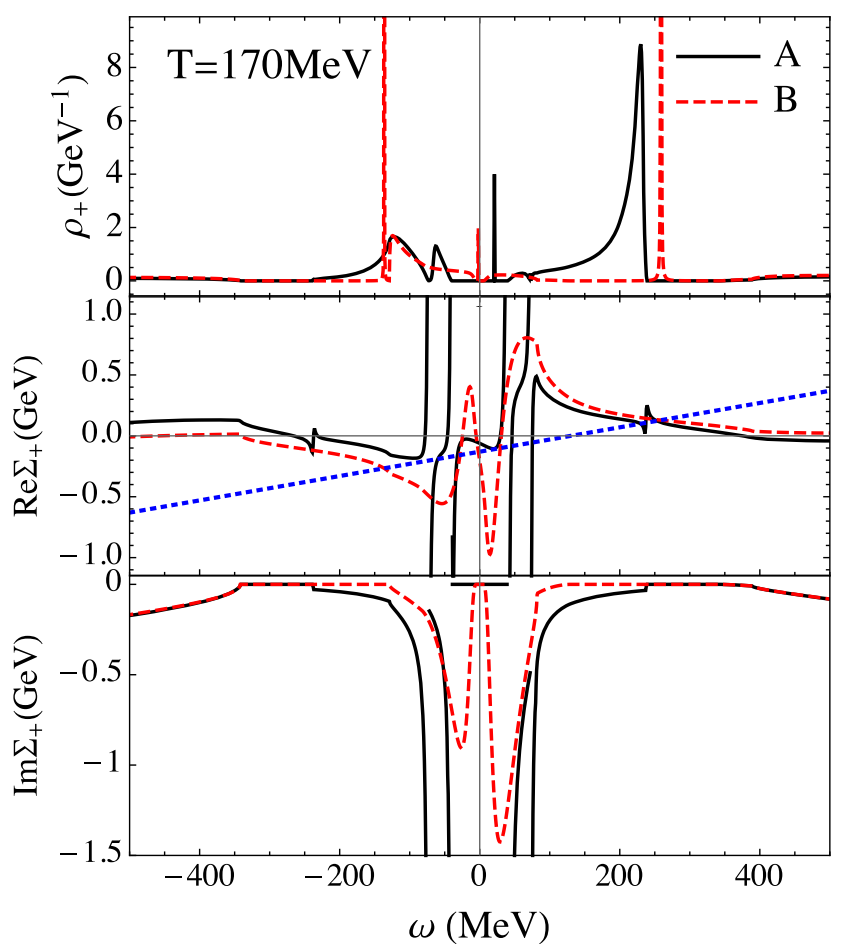

FIG. 5. Quark spectral function at $T=170 \mathrm{MeV}$ : from top to bottom are the spectral function and real and imaginary parts of the quark self-energy. The black solid line stands for truncation A, while red dashed line stands for truncation B. The blue dotted line represents $\omega-m_{f}$, with $m_{f}=130 \mathrm{MeV}$.

imaginary part is nonzero but finite when processes II and III are allowed. The real part has five quasipoles, and at three of them, the imaginary part is small enough to give a peak in the spectral function. When processes II and III are allowed, the imaginary part is large and gives only small bumps in the spectral function; see the red dashed lines in Fig. 5. The spectral function presents a three-peak structure in truncation $\mathrm{B}$, with one peak at the origin, and two quasipoles where the imaginary part is small. This is the typical structure at $T \sim m_{b}$ and has been discussed in depth in Ref. [12]. The Landau damping, which causes peaks in the imaginary part, is essential in the three-peak structure in the spectral function. In truncation A, the spectral function also presents a peak at small $\omega$ but not at the exact origin; namely, instead of a zero mode, we have a soft mode in truncation $\mathrm{A}$, which also arises from the Landau damping effect.

Finally, we analyze the spectral function in both truncations at $T=300 \mathrm{MeV}$, where the system has reached the chiral restored phase, with $m_{f}=24 \mathrm{MeV}$, and degenerate meson mass $m_{\sigma} \approx m_{\pi}=490 \mathrm{MeV}$. The result is presented in Fig. 6. For truncation A, the threshold of each channel is presented in the last figure in Fig. 3. There is a large area where channels II and III are forbidden, leading to $\operatorname{Im} \Sigma_{+}=$ 0 at small energy. Channels II and III both have points where $\pm E_{\phi, k^{*}}^{\prime} \mp E_{\psi, k^{*}}^{\prime}=0$, where the imaginary part 


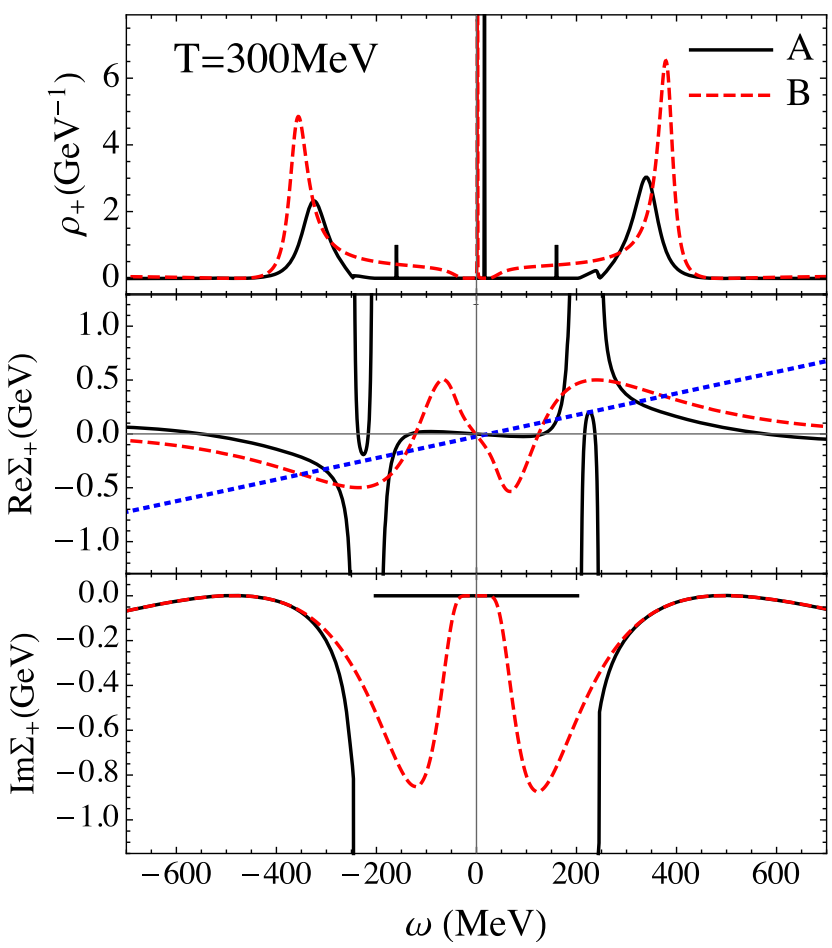

FIG. 6. Quark spectral function at $T=300 \mathrm{MeV}$ : from top to bottom are the spectral function and real and imaginary parts of the quark self-energy. The black solid line stands for truncation A, while red dashed line stands for truncation B. The blue dotted line represents $\omega-m_{f}$, with $m_{f}=24 \mathrm{MeV}$.

diverges. The real part of the inverse propagator has seven quasipoles, at two of which the imaginary parts are too large to give a peak at $\omega=10, \pm 160 \mathrm{MeV}$. For the three quasipoles at low energy, the imaginary part is small and gives three peaks. For the two quasipoles at large $\omega$, the Landau damping effect gives two bumps in the spectral function, while in truncation B, the Landau damping effect again gives two peaks in the imaginary part of the selfenergy, and an oscillation in the real part. $\omega-m_{f}-$ $\operatorname{Re} \Sigma_{+}(\omega)=0$ has five quasipoles, and $\operatorname{Im} \Sigma_{+}$has relatively large values at four of the quasipoles, leading to two peaks with finite width, and a delta peak at the origin.

Two scales are of crucial importance in the structure of the fermion spectral function: $m_{f} / m_{b}$ and $T / m_{b}$. The threepeak structure is most obvious when $m_{f} / m_{b} \ll 1$ and $T / m_{b} \sim 1$. When these two factors are approached, the peaks become higher and sharper. For the chiral crossover, $T / m_{b} \sim 1$ takes place around the critical temperature, where we observe three sharp peaks in truncation B. For high temperature $T / m_{b} \approx 0.61, m_{f} / m_{b} \ll 1$ is satisfied; thus the fermion is almost massless, and the three-peak structure is also obvious but with finite width. In truncation $\mathrm{B}$, the appearance of a zero mode can be demonstrated by analyzing the real and imaginary parts of self-energy at $\omega=0$. In the case of finite fermion mass, one always has $\operatorname{Im} \Sigma_{+}(\omega=0)=0$, and $\operatorname{Re} \Sigma_{+}(\omega)$ can be exactly calculated to give a quasipole very close to the origin; thus, a peak will appear at the origin. In truncation $\mathrm{A}$, the multipeak structure is also observed as $T / m_{b}$ approaches unit. However, the zero mode becomes a soft mode, in which the quasipole is slightly away from the origin. The disappearance of this zero mode results from the limited scale of momentum in the propagator and the scale dependence of the meson masses. One may expect that with larger $\Lambda$ the soft mode would be closer to the origin.

\section{SUMMARY}

We investigate the spectral function of the quark in two truncations in a quark-meson model with the functional renormalization group. In both truncations, we first solve the flow equation of the effective potential and find out the temperature and scale dependence of the fermion and meson masses. In truncation $\mathrm{A}$, we take the scaledependent masses in step 1 as the input and evolve the flow equation of the two-point function; in truncation B, we take the masses in the infrared as input and calculate the oneloop self-energy. After the analytical continuation, we have the spectral function.

When one consistently integrates the flow equation of the two-point function, the RG-scale dependence of the energy thresholds of decay and creation channels leads to van Hove singularities at finite temperature, where Landau damping plays an important role. This singularity leads to divergence in both the real and imaginary parts. Another feature is that at high temperature the Landau damping is forbidden at low energy, leading to zero imaginary parts and several peaks in the low-energy area.

In comparison, when directly calculating the one-loop self-energy, one gets a three-peak structure when the temperature rises and becomes comparable to meson mass. The spectral function has a peak at the origin, namely, a fermion zero mode, and the other peaks come from the Landau damping effect.

Our work presents a first calculation of the finitetemperature quark spectral function and supports the quasiparticle picture of quarks around the critical temperature.

\section{ACKNOWLEDGMENTS}

The work is supported by the National Natural Science Foundation of China (Grants No. 11335005, No. 11575093, and No. 11775123), MOST (Grants No. 2013CB922000 and No. 2014CB845400).

Note Added.-Recently, we became aware of the work by R. A. Tripolt et al. [28], in which the fermion spectral function at zero temperature was investigated.

\section{APPENDIX: LOOP FUNCTIONS}

With the boson and fermion occupation numbers and their derivatives, 


$$
\begin{array}{rlrl}
n_{B}(x) & =\frac{1}{e^{x / T}-1}, & & n_{F}(x)=\frac{1}{e^{x / T}+1}, \\
n_{B}^{\prime}(x)=\frac{d n_{B}(x)}{d x}, & n_{F}^{\prime}(x)=\frac{d n_{F}(x)}{d x},
\end{array}
$$

the loop functions $J_{\phi}$ and $J_{\psi}$ in the flow equation for effective potential are explicitly expressed as

$$
\begin{aligned}
J_{\phi} & =\frac{k^{4}}{3 \pi^{2}} \frac{1+2 n_{B}\left(E_{\phi}\right)}{2 E_{\phi}}, \\
J_{\psi} & =\frac{k^{4}}{3 \pi^{2}} \frac{1-n_{F}\left(E_{\psi}-\mu\right)-n_{F}\left(E_{\psi}+\mu\right)}{E_{\psi}} .
\end{aligned}
$$

The threshold function $J_{\psi \sigma}^{S}, J_{\sigma \psi}^{S}, J_{\psi \pi}^{P S}, J_{\pi \psi}^{P S}$ in the two-point function can be obtained by taking derivatives with respect to the corresponding energy,

$$
\begin{aligned}
& J_{\psi \sigma}^{S}\left(i p_{0}\right)=-\frac{1}{2 E_{\psi}} \frac{\partial}{\partial E_{\psi}} J^{S}\left(i p_{0}\right), \\
& J_{\sigma \psi}^{S}\left(i p_{0}\right)=-\frac{1}{2 E_{\sigma}} \frac{\partial}{\partial E_{\sigma}} J^{S}\left(i p_{0}\right), \\
& J_{\psi \pi}^{P S}\left(i p_{0}\right)=-\frac{1}{2 E_{\psi}} \frac{\partial}{\partial E_{\psi}} J^{P S}\left(i p_{0}\right), \\
& J_{\pi \psi}^{P S}\left(i p_{0}\right)=-\frac{1}{2 E_{\pi}} \frac{\partial}{\partial E_{\pi}} J^{P S}\left(i p_{0}\right) .
\end{aligned}
$$

After the analytical continuation, $J^{S}$ in Minkovski space is

$$
\begin{aligned}
J^{S}(\omega)= & -\frac{k^{4}}{12 \pi^{2} E_{\phi}}\left\{\frac{1}{\omega+\mu+E_{\phi}+E_{\psi}+i \eta}\left(1-\frac{m_{f}}{E_{\psi}}\right)\left(1+n_{B}\left(E_{\phi}\right)-n_{F}\left(E_{\psi}+\mu\right)\right)\right. \\
& +\frac{1}{\omega+\mu+E_{\phi}-E_{\psi}+i \eta}\left(1+\frac{m_{f}}{E_{\psi}}\right)\left(n_{B}\left(E_{\phi}\right)+n_{F}\left(E_{\psi}-\mu\right)\right) \\
& +\frac{1}{\omega+\mu-E_{\phi}+E_{\psi}+i \eta}\left(1-\frac{m_{f}}{E_{\psi}}\right)\left(n_{B}\left(E_{\phi}\right)+n_{F}\left(E_{\psi}+\mu\right)\right) \\
& \left.+\frac{1}{\omega+\mu-E_{\phi}-E_{\psi}+i \eta}\left(1+\frac{m_{f}}{E_{\psi}}\right)\left(1+n_{B}\left(E_{\phi}\right)-n_{F}\left(E_{\psi}-\mu\right)\right)\right\}
\end{aligned}
$$

Making substitution by $m_{f} \rightarrow-m_{f}$ and $E_{\sigma} \rightarrow E_{\pi}$, then we can get the threshold for pseudoscalar channel $J^{P S}(\omega)$. The real part is given by the principle value, while the imaginary part of the threshold function is then

$$
\begin{aligned}
\operatorname{Im} J^{S}(\omega)= & \frac{k^{4}}{12 \pi E_{\phi}}\left\{\delta\left(\omega+\mu+E_{\phi}+E_{\psi}\right)\left(1-\frac{m_{f}}{E_{\psi}}\right)\left(1+n_{B}\left(E_{\phi}\right)-n_{F}\left(E_{\psi}+\mu\right)\right)\right. \\
& +\delta\left(\omega+\mu+E_{\phi}-E_{\psi}\right)\left(1+\frac{m_{f}}{E_{\psi}}\right)\left(n_{B}\left(E_{\phi}\right)+n_{F}\left(E_{\psi}-\mu\right)\right) \\
& +\delta\left(\omega+\mu-E_{\phi}+E_{\psi}\right)\left(1-\frac{m_{f}}{E_{\psi}}\right)\left(n_{B}\left(E_{\phi}\right)+n_{F}\left(E_{\psi}+\mu\right)\right) \\
& \left.+\delta\left(\omega+\mu-E_{\phi}-E_{\psi}\right)\left(1+\frac{m_{f}}{E_{\psi}}\right)\left(1+n_{B}\left(E_{\phi}\right)-n_{F}\left(E_{\psi}-\mu\right)\right)\right\} .
\end{aligned}
$$

Following Eq. (A3), one has the imaginary part of the threshold functions $\operatorname{Im} J_{\psi \phi}^{S}, \operatorname{Im} J_{\phi \psi}^{S}$ for the scalar channel,

$$
\begin{aligned}
\operatorname{Im} J_{\psi \phi}^{S}(\omega)= & -\frac{k^{4}}{24 \pi E_{\phi} E_{\psi}}\left\{\delta^{\prime}\left(\omega+\mu+E_{\phi}+E_{\psi}\right)\left(1-\frac{m_{f}}{E_{\psi}}\right)\left(1+n_{B}\left(E_{\phi}\right)-n_{F}\left(E_{\psi}+\mu\right)\right)\right. \\
& -\delta^{\prime}\left(\omega+\mu+E_{\phi}-E_{\psi}\right)\left(1+\frac{m_{f}}{E_{\psi}}\right)\left(n_{B}\left(E_{\phi}\right)+n_{F}\left(E_{\psi}-\mu\right)\right) \\
& +\delta^{\prime}\left(\omega+\mu-E_{\phi}+E_{\psi}\right)\left(1-\frac{m_{f}}{E_{\psi}}\right)\left(n_{B}\left(E_{\phi}\right)+n_{F}\left(E_{\psi}+\mu\right)\right) \\
& \left.-\delta^{\prime}\left(\omega+\mu-E_{\phi}-E_{\psi}\right)\left(1+\frac{m_{f}}{E_{\psi}}\right)\left(1+n_{B}\left(E_{\phi}\right)-n_{F}\left(E_{\psi}-\mu\right)\right)\right\}
\end{aligned}
$$




$$
\begin{aligned}
& -\frac{k^{4} m_{f}}{24 \pi E_{\phi} E_{\psi}^{3}}\left\{\delta\left(\omega+\mu+E_{\phi}+E_{\psi}\right)\left[1+n_{B}\left(E_{\phi}\right)-n_{F}\left(E_{\psi}+\mu\right)-E_{\psi}\left(\frac{E_{\psi}}{m_{f}}-1\right) n_{F}^{\prime}\left(E_{\psi}+\mu\right)\right]\right. \\
& -\delta\left(\omega+\mu+E_{\phi}-E_{\psi}\right)\left[n_{B}\left(E_{\phi}\right)+n_{F}\left(E_{\psi}-\mu\right)-E_{\psi}\left(\frac{E_{\psi}}{m_{f}}+1\right) n_{F}^{\prime}\left(E_{\psi}-\mu\right)\right] \\
& +\delta\left(\omega+\mu-E_{\phi}+E_{\psi}\right)\left[n_{B}\left(E_{\phi}\right)+n_{F}\left(E_{\psi}+\mu\right)+E_{\psi}\left(\frac{E_{\psi}}{m_{f}}-1\right) n_{F}^{\prime}\left(E_{\psi}+\mu\right)\right] \\
& \left.-\delta\left(\omega+\mu-E_{\phi}-E_{\psi}\right)\left[1+n_{B}\left(E_{\phi}\right)-n_{F}\left(E_{\psi}-\mu\right)+E_{\psi}\left(\frac{E_{\psi}}{m_{f}}+1\right) n_{F}^{\prime}\left(E_{\psi}-\mu\right)\right]\right\},
\end{aligned}
$$

and

$$
\begin{aligned}
\operatorname{Im} J_{\phi \psi}^{S}(\omega)= & -\frac{k^{4}}{24 \pi E_{\phi}^{2}}\left\{\delta^{\prime}\left(\omega+\mu+E_{\phi}+E_{\psi}\right)\left(1-\frac{m_{f}}{E_{\psi}}\right)\left(1+n_{B}\left(E_{\phi}\right)-n_{F}\left(E_{\psi}+\mu\right)\right)\right. \\
& +\delta^{\prime}\left(\omega+\mu+E_{\phi}-E_{\psi}\right)\left(1+\frac{m_{f}}{E_{\psi}}\right)\left(n_{B}\left(E_{\phi}\right)+n_{F}\left(E_{\psi}-\mu\right)\right) \\
& -\delta^{\prime}\left(\omega+\mu-E_{\phi}+E_{\psi}\right)\left(1-\frac{m_{f}}{E_{\psi}}\right)\left(n_{B}\left(E_{\phi}\right)+n_{F}\left(E_{\psi}+\mu\right)\right) \\
& \left.-\delta^{\prime}\left(\omega+\mu-E_{\phi}-E_{\psi}\right)\left(1+\frac{m_{f}}{E_{\psi}}\right)\left(1+n_{B}\left(E_{\phi}\right)-n_{F}\left(E_{\psi}-\mu\right)\right)\right\} \\
& +\frac{k^{4}}{24 \pi E_{\phi}^{3}}\left\{\delta\left(\omega+\mu+E_{\phi}+E_{\psi}\right)\left(1-\frac{m_{f}}{E_{\psi}}\right)\left(1+n_{B}\left(E_{\phi}\right)-n_{F}\left(E_{\psi}+\mu\right)-E_{\phi} n_{B}^{\prime}\left(E_{\phi}\right)\right)\right. \\
& +\delta\left(\omega+\mu+E_{\phi}-E_{\psi}\right)\left(1+\frac{m_{f}}{E_{\psi}}\right)\left(n_{B}\left(E_{\phi}\right)+n_{F}\left(E_{\psi}-\mu\right)-E_{\phi} n_{B}^{\prime}\left(E_{\phi}\right)\right) \\
& +\delta\left(\omega+\mu-E_{\phi}+E_{\psi}\right)\left(1-\frac{m_{f}}{E_{\psi}}\right)\left(n_{B}\left(E_{\phi}\right)+n_{F}\left(E_{\psi}+\mu\right)-E_{\phi} n_{B}^{\prime}\left(E_{\phi}\right)\right) \\
& \left.+\delta\left(\omega+\mu-E_{\phi}-E_{\psi}\right)\left(1+\frac{m_{f}}{E_{\psi}}\right)\left(1+n_{B}\left(E_{\phi}\right)-n_{F}\left(E_{\psi}-\mu\right)-E_{\phi} n_{B}^{\prime}\left(E_{\phi}\right)\right)\right\} .
\end{aligned}
$$

[1] J. Adams et al. (STAR Collaboration), Nucl. Phys. A757, 102 (2005).

[2] K. Adcox et al. (PHENIX Collaboration), Nucl. Phys. A757, 184 (2005).

[3] F. Karsch and M. Kitazawa, Phys. Lett. B 658, 45 (2007).

[4] F. Karsch and M. Kitazawa, Phys. Rev. D 80, 056001 (2009).

[5] O. Kaczmarek, F. Karsch, M. Kitazawa, and W. Soldner, Phys. Rev. D 86, 036006 (2012).

[6] M. Harada and Y. Nemoto, Phys. Rev. D 78, 014004 (2008).

[7] J. A. Mueller, C. S. Fischer, and D. Nickel, Eur. Phys. J. C 70, 1037 (2010).

[8] R. D. Pisarski, Phys. Rev. Lett. 63, 1129 (1989).

[9] E. Braaten and R. D. Pisarski, Nucl. Phys. B337, 569 (1990).
[10] N. Su and K. Tywoniuk, Phys. Rev. Lett. 114, 161601 (2015).

[11] M. Kitazawa, T. Kunihiro, and Y. Nemoto, Phys. Lett. B 633, 269 (2006).

[12] M. Kitazawa, T. Kunihiro, K. Mitsutani, and Y. Nemoto, Phys. Rev. D 77, 045034 (2008).

[13] M. Kitazawa, T. Kunihiro, and Y. Nemoto, Prog. Theor. Phys. 117, 103 (2007).

[14] J. Berges, N. Tetradis, and C. Wetterich, Phys. Rep. 363, 223 (2002).

[15] J. Polonyi, Central Eur. J. Phys. 1, 1 (2003).

[16] J. M. Pawlowski, Ann. Phys. (Amsterdam) 322, 2831 (2007).

[17] H. Gies, Lect. Notes Phys. 852, 287 (2012).

[18] P. Kopietz, L. Bartosch, and F. Schtz, Lect. Notes Phys. 798, 1 (2010). 
[19] J. Braun, J. Phys. G 39, 033001 (2012).

[20] K. Kamikado, N. Strodthoff, L. von Smekal, and J. Wambach, Eur. Phys. J. C 74, 2806 (2014).

[21] J. M. Pawlowski and N. Strodthoff, Phys. Rev. D 92, 094009 (2015).

[22] N. Strodthoff, Phys. Rev. D 95, 076002 (2017).

[23] R. A. Tripolt, N. Strodthoff, L. von Smekal, and J. Wambach, Phys. Rev. D 89, 034010 (2014).

[24] R. A. Tripolt, L. von Smekal, and J. Wambach, Phys. Rev. D 90, 074031 (2014).

[25] C. Jung, F. Rennecke, R. A. Tripolt, L. von Smekal, and J. Wambach, Phys. Rev. D 95, 036020 (2017).

[26] R. A. Tripolt, L. von Smekal, and J. Wambach, Int. J. Mod. Phys. E 26, 1740028 (2017).

[27] T. Yokota, T. Kunihiro, and K. Morita, Prog. Theor. Exp. Phys. 2016, 073D01 (2016).

[28] R. A. Tripolt, J. Weyrich, L. von Smekal, and J. Wambach, Phys. Rev. D 98, 094002 (2018).
[29] Z. Wang and P. Zhuang, Phys. Rev. D 96, 014006 (2017).

[30] D. U. Jungnickel and C. Wetterich, Phys. Rev. D 53, 5142 (1996).

[31] B. J. Schaefer and J. Wambach, Nucl. Phys. A757, 479 (2005).

[32] J. M. Pawlowski and F. Rennecke, Phys. Rev. D 90, 076002 (2014).

[33] D. F. Litim, Phys. Lett. B 486, 92 (2000).

[34] D. F. Litim, Phys. Rev. D 64, 105007 (2001).

[35] L. Van Hove, Phys. Rev. 89, 1189 (1953).

[36] M. G. Mustafa, A. Schafer, and M. H. Thoma, Phys. Rev. C 61, 024902 (1999).

[37] A. Peshier and M.H. Thoma, Phys. Rev. Lett. 84, 841 (2000).

[38] F. Karsch, M. G. Mustafa, and M. H. Thoma, Phys. Lett. B 497, 249 (2001).

[39] M. G. Mustafa and M. H. Thoma, Pramana 60, 711 (2003). 